\title{
Broadband circular polarizer based on high-contrast gratings
}

\author{
Mehmet Mutlu,* Ahmet E. Akosman, and Ekmel Ozbay \\ Department of Electrical and Electronics Engineering, Nanotechnology Research Center, \\ Bilkent University, 06800 Ankara, Turkey \\ *Corresponding author: mutlu@ee.bilkent.edu.tr
}

Received March 19, 2012; accepted April 6, 2012;

posted April 10, 2012 (Doc. ID 165012); published May 30, 2012

\begin{abstract}
A circular polarizer, which is composed of periodic and two-dimensional dielectric high-contrast gratings, is designed theoretically such that a unity conversion efficiency is achieved at $\lambda_{0}=1.55 \mu \mathrm{m}$. The operation is obtained by the achievement of the simultaneous unity transmission of transverse magnetic and transverse electric waves with a phase difference of $\pi / 2$, meaning that an optimized geometrical anisotropy is accomplished. By the utilization of the rigorous coupled-wave analysis and finite-difference time-domain methods, it is shown that a percent bandwidth of $\sim 50 \%$ can be achieved when the operation bandwidth is defined as the wavelengths for which the conversion efficiency exceeds 0.9. (C) 2012 Optical Society of America
\end{abstract}

OCIS codes: $\quad 050.2770,260.5430,050.6624$.

Binary high-contrast gratings (HCGs) with subwavelength periodicities have recently attracted significant interest mostly because of their broadband and diffraction-free high-reflectivity regimes [1,2]. Among the efforts based on the extraordinary properties of HCGs, vertical-cavity surface-emitting lasers [3], ultralow loss

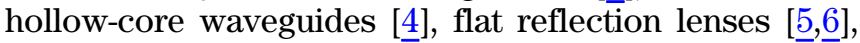
broadband polarization independent reflectors [7], polarizing beam splitters [8], unidirectional transmission devices [9], and beam-steering structures [10] should be mentioned. The utilization of HCGs provides a geometrical design flexibility for the achievement of the desired transmission and reflection characteristics along with the small footprint and reasonable fabrication requirements.

In this letter, we study the potential of a broadband circular polarizer by the optimization of the transverse magnetic (TM) and transverse electric (TE) polarization complex transmission coefficients. The proposed design is depicted in Fig. 1 and composed of periodic twodimensional (infinite in the $y$ direction) binary gratings. For a realistic design, the substrate filling region III is selected as silicon dioxide $\left(\mathrm{SiO}_{2}\right)$ and, because of impedance matching concerns for the achievement of unity transmission, region I and the grooves in region II are also filled with $\mathrm{SiO}_{2}$. The high-index material that constitutes the ridges is selected as silicon $(\mathrm{Si})$. In the practical realization stage, the infinite medium effects in regions I and III can be approximately achieved by the deposition of an antireflective coating and the utilization of a very thick $(\sim 1 \mathrm{~mm}) \mathrm{SiO}_{2}$ substrate, respectively.

In adopting the theoretical analysis given in [11], the condition for the unit transmittance of a TM polarized incident wave can be written as follows:

$$
\left|\sum_{m}\left(a_{m}-a_{m}^{\rho}\right) \Lambda^{-1} \int_{0}^{\Lambda} h_{y, m}^{\text {in }}(x) \mathrm{d} x\right|=1,
$$

where $a_{m}$ and $\alpha_{m}^{\rho}$ are the coefficients of the $+z$ and $-z$ propagating field components of the $m$ th waveguide mode, respectively, and $h_{y, m}^{\text {in }}$ represents the lateral magnetic field distribution inside region II. The condition given in Eq. (1) implies that the magnitude of the sum of the transmission coefficients for each mode should add up to unity. It is noteworthy that the obtaining of identical phases for the transmission coefficients of the individual modes is not a requirement. Benefiting from the duality, the unit transmittance condition for TE waves can be written by replacing $h_{y, m}^{\text {in }}$ in Eq. (1) with $h_{x, m}^{\text {in }}$.

To enable the obtaining of a structure that acts as a circular polarizer for linearly polarized incident plane waves with a polarization angle of $\pi / 4$, Eq. (1) must be simultaneously satisfied for both TM and TE waves. Furthermore, the phase difference between the TM and TE transmission coefficients must be $\pm \pi / 2$. This phase condition implies the following equation:

$$
\angle\left(\sum_{m} \mathbf{H}_{0, m}^{\mathrm{TM}} A^{\mathrm{TM}}\right)-\angle\left(\sum_{m} \mathbf{H}_{0, m}^{\mathrm{TE}} A^{\mathrm{TE}}\right)= \pm \pi / 2
$$

where $A=\left(a_{m}-a_{m}^{\rho}\right)$ and $\mathbf{H}_{0, m}^{\mathrm{TM}}$ is given as follows:

$$
\mathbf{H}_{0, m}^{\mathrm{TM}}=\Lambda^{-1} \int_{0}^{\Lambda} h_{y, m}^{\mathrm{in}}(x) \mathrm{d} x .
$$

In the calculation of $\mathbf{H}_{0, m}^{\mathrm{TE}}, h_{y, m}^{\mathrm{in}}$ in Eq. (3) should be replaced by $h_{x, m}^{\mathrm{in}}$. Furthermore, one can show that the longitudinal wavenumber inside the grating region, $\beta_{m}$, is given by

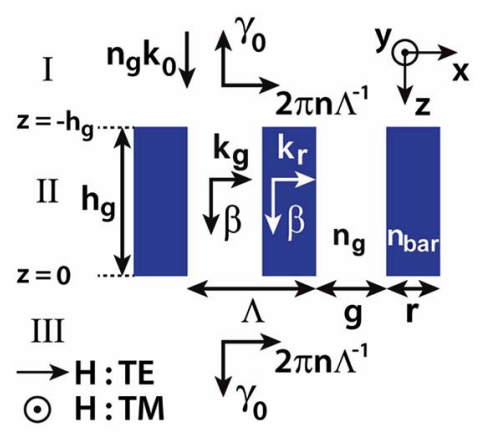

Fig. 1. (Color online) Geometrical description of the HCG circular polarizer. The dark regions indicate the presence of $\mathrm{Si}$, whereas the white regions are $\mathrm{SiO}_{2}$. 


$$
\beta_{m}^{2}=\left(2 \pi n_{g} / \lambda_{0}\right)^{2}-k_{g, m}^{2}=\left(2 \pi n_{\mathrm{bar}} / \lambda_{0}\right)^{2}-k_{r, m}^{2},
$$

where $\lambda_{0}$ is the free-space wavelength and $k_{g, m}$ and $k_{r, m}$ are the lateral wavenumbers of the $m$ th waveguide mode inside the grooves and the ridges, respectively. The dispersion relation between $k_{g}$ and $k_{r}$ for TM waves is given as follows:

$$
n_{\mathrm{bar}}^{-2} k_{r, m} \tan \left(k_{r, m} r / 2\right)=-n_{g}^{-2} k_{g, m} \tan \left(k_{g, m} g / 2\right) .
$$

For TE waves, the dispersion relation is given by

$$
k_{r, m} \tan \left(k_{r, m} r / 2\right)=-k_{g, m} \tan \left(k_{g, m} g / 2\right) .
$$

As a result of the different dispersion relations, the longitudinal and lateral wavenumbers of the TM and TE modes can be different. At this point, we invoke a two-mode approximation such that the allowed values of $m$ are 0 and 1 . The corresponding $k_{g}, k_{r}$, and $\beta$ values for a certain $h_{g}, r, g$, and $\Lambda$ geometrical parameter set can be determined by the simultaneous solution of Eqs. (4) and (5) for TM waves and Eqs. (4) and (6) for TE waves. The wavelength of interest $\left(\lambda_{0}\right)$ is selected as $1.55 \mu \mathrm{m} . n_{\mathrm{bar}}$ and $n_{g}$ are set to 3.48 and 1.47 , respectively, which correspond to the refractive indices of $\mathrm{Si}$ and $\mathrm{SiO}_{2}$ at this frequency. Thereafter, with the usage of a custom parametric optimization code, the geometrical parameters that satisfy Eqs. (1) (for TM and TE waves simultaneously) and (2) are obtained as $r=160 \mathrm{~nm}, g=$ $220 \mathrm{~nm}, \Lambda=380 \mathrm{~nm}$, and $h_{g}=550 \mathrm{~nm}$.

According to the given geometrical parameters, for TM waves, the lateral wavenumbers inside region II are calculated as $k_{g, 0}^{2}=-4.2 / \Lambda^{2}, k_{r, 0}^{2}=19.43 / \Lambda^{2}$ and $k_{g, 1}^{2}=$ $31.3 / \Lambda^{2}, k_{r, 1}^{2}=54.9 / \Lambda^{2}$. In a waveguide, the mode cutoff corresponds to $\beta_{m}=0$ and accordingly, in this system, the mode cutoff condition, which is derived from Eq. (4), is given by $k_{r}^{2}=\left(n_{\mathrm{bar}} / n_{g}\right)^{2} k_{g}^{2}$. For larger $k_{r}$, the resulting $\beta_{m}$ values are negative, which implies that the corresponding waveguide mode is exponentially decaying in the propagation direction $( \pm z)$. Thus, the first TM mode is a propagating waveguide mode, whereas the second one corresponds to an exponentially decaying mode.

By performing a similar analysis for TE waves, the lateral wavenumbers inside the grating region are obtained as $k_{g, 0}^{2}=-13.1 / \Lambda^{2}, k_{r, 0}^{2}=10.48 / \Lambda^{2}$ and $k_{g, 1}^{2}=30.2 / \Lambda^{2}$, $k_{r, 1}^{2}=53.8 / \Lambda^{2}$. The lateral wavenumbers in the TE case again imply that the first mode and the second mode are propagating and evanescent modes, respectively.

The longitudinal wavenumber of the $n$th order diffracted wave in transmission and reflection is denoted by $\gamma_{n}$ and defined as $\gamma_{n}^{2}=\left(2 \pi n_{g}\right)^{2}\left(\lambda_{0}^{-2}-n^{2} \Lambda^{-2}\right)$. Since $\lambda_{0} / \Lambda=4.1$ in the proposed design, only the zeroth diffraction order is propagating, whereas all of the higher orders are evanescent, which results in the fact that the reflected and transmitted waves are plane waves propagating in the $-z$ and $+z$ directions, respectively.

The magnitudes of the corresponding field transmission coefficients are calculated for the TM and TE cases as 0.988 and 0.936 , respectively. The phase difference between these two coefficients is defined as $\angle T_{\mathrm{TM}}-\angle T_{\mathrm{TE}}$, and its value is obtained as $92^{\circ}$, which is desired for the obtaining of a circularly polarized wave at the output interface. Accordingly, it can be deduced that a circularly polarized wave should be transmitted if the structure is illuminated by a plane wave that is linearly polarized at an angle of $\pi / 4$, meaning that the electric field components that correspond to the TE and TM polarizations have equal magnitudes and are also in-phase. The magnetic field distributions inside the region II obtained under the two-mode approximation for TM and TE waves are shown in Fig. 2. The unequal lateral and longitudinal wavenumbers for the TE and TM modes, which also lead to unequal $a_{m}$ and $a_{m}^{\rho}$ values along with unequal $h_{y, m}^{\text {in }}$ and $h_{x, m}^{\mathrm{in}}$ distributions, result in significantly different field distributions inside the periodic waveguide. For the further details of the calculation of the transmission coefficients and the field distributions, one can refer to [11], which provides a detailed theoretical analysis for vertical binary gratings.

To verify the validity of the results obtained via the theoretical model, we employ the rigorous coupled-wave analysis (RCWA) [12]. Using this method, between the free-space wavelengths of $1 \mu \mathrm{m}$ and $3.5 \mu \mathrm{m}$, the magnitudes of the TM and TE transmission coefficients and their phase difference are determined, and these results are shown in Fig. 3. Thereafter, the circular conversion coefficients, which indicate the amplitudes of the righthand circularly polarized $(\mathrm{RCP},+)$ and left-hand circularly polarized (LCP, -) waves at the output interface, for an incident wave that is linearly polarized at an angle of $\pi / 4$ in the $x y$-plane can be calculated with a simple transformation from the linear base to the circular base as $C_{ \pm}=0.5\left(T_{\mathrm{TM}} \mp i T_{\mathrm{TE}}\right)[\underline{13}]$. At this point, we define a conversion efficiency to characterize the performance of the proposed structure, which is denoted by $C_{\text {eff }}$ and can be given as follows:

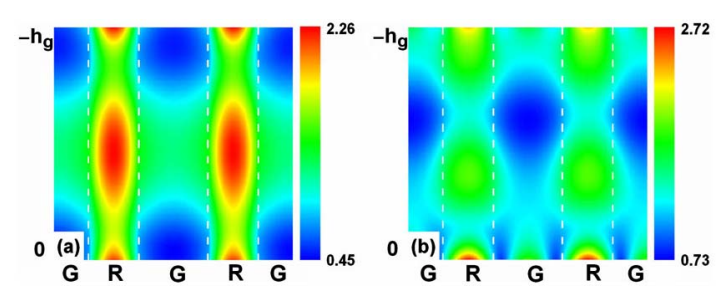

Fig. 2. (Color online) Normalized magnetic field distributions at $\lambda_{0}=1.55 \mu \mathrm{m}$ inside region II, (a) $\left|H_{y}\right| /\left|H_{0}\right|$ and (b) $\left|H_{x}\right| /\left|H_{0}\right|$ for TM and TE waves, respectively. The distributions are obtained under the two-mode approximation. Grooves and ridges are separated by the white dashed lines and denoted by $\mathrm{G}$ and $R$, respectively.
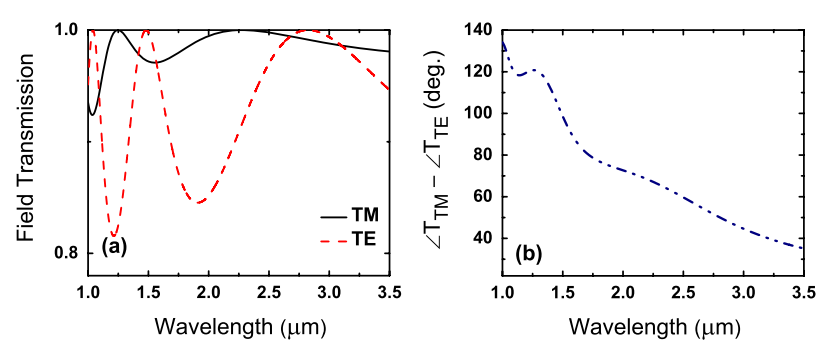

Fig. 3. (Color online) (a) Field transmission coefficients for TM and TE waves, and (b) phase difference between the transmission coefficients. 

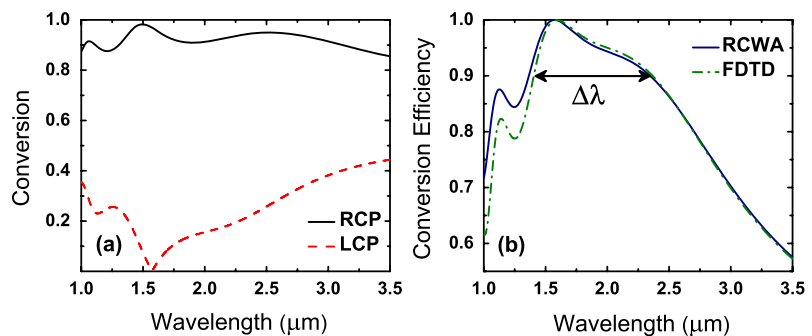

Fig. 4. (Color online) (a) Circular conversion coefficients obtained using the RCWA and (b) the conversion efficiencies obtained from the RCWA and FDTD. The wavelength range for the FDTD result satisfying the 0.9 efficiency threshold is denoted by $\Delta \lambda$.

$$
C_{\text {eff }}=\frac{\left|C_{+}\right|^{2}-\left|C_{-}\right|^{2}}{\left|C_{+}\right|^{2}+\left|C_{-}\right|^{2}} \text {. }
$$

To incorporate the effects arising because of the material dispersion of $\mathrm{Si}$ and $\mathrm{SiO}_{2}$, we perform finitedifference time-domain (FDTD) simulations using FDTD Solutions (Lumerical, Inc.), where the refractive index data given in [14] is utilized. Figure 4 shows the circular conversion coefficients obtained from the RCWA along with the conversion efficiencies calculated using the RCWA and FDTD methods. The RCWA method suggests that a conversion efficiency that is larger than 0.9 is achieved between the wavelengths of $1.36 \mu \mathrm{m}$ and $2.36 \mu \mathrm{m}$. According to the FDTD results, where the material dispersion is taken into account properly, the 0.9 threshold is achieved between $1.4 \mu \mathrm{m}$ and $2.36 \mu \mathrm{m}$. As a consequence of the strongly pronounced material dispersion effects for $\mathrm{Si}$ for wavelengths smaller than $1.5 \mu \mathrm{m}$, a slightly narrower bandwidth is achieved. If the percent bandwidth is defined as follows [15]:

$$
\mathrm{BW} \%=200 \% \frac{\lambda_{H} / \lambda_{L}-1}{\lambda_{H} / \lambda_{L}+1},
$$

where $\lambda_{H}$ and $\lambda_{L}$ are the higher and lower corner wavelengths, respectively, a BW\% of $54 \%$ and $51 \%$ is achieved using the RCWA and FDTD methods, respectively. Despite causing an observable modification in the conversion efficiency spectrum, the involvement of the material dispersion effects does not significantly modify the percent bandwidth, which shows that the proposed structure in the present study can be practically realizable.

To summarize, we have designed an optimized twodimensional HCG such that the transmitted wave is circularly polarized if the structure is illuminated by a plane wave with a polarization plane angle of $\pi / 4$. It has numerically been shown that a conversion efficiency that is larger than 0.9 can be achieved within a $51 \%$ bandwidth. Some possible application areas of the proposed structure can be listed as nanoantenna and laser applications, remote sensors, and liquid crystal displays. As future research, the fabrication and the experimental characterization of the proposed structure can be performed.

This work is supported by the projects DPT-HAMIT (Turkish Republic Ministry of Development), ESF-EPIGRAT (European Science Foundation), EU-N4E (European Union), NATO-SET-181 (North Atlantic Treaty Organization), and The Scientific and Technological Research Council of Turkey under Projects No. 107A004, No. 107A012, and No. 109E301. One of the authors (E.O.) also acknowledges partial support from the Turkish Academy of Sciences.

\section{References}

1. C. Mateus, M. Huang, Y. Deng, A. Neureuther, and C. Chang-Hasnain, IEEE Photon. Technol. Lett. 16, 518 (2004).

2. C. Mateus, M. Huang, L. Chen, C. Chang-Hasnain, and Y. Suzuki, IEEE Photon. Technol. Lett. 16, 1676 (2004).

3. M. C. Y. Huang, Y. Zhou, and C. J. Chang-Hasnain, Nat. Photon. 1, 119 (2007).

4. Y. Zhou, V. Karagodsky, B. Pesala, F. G. Sedgwick, and C. J. Chang-Hasnain, Opt. Express 17, 1508 (2009).

5. F. Lu, F. G. Sedgwick, V. Karagodsky, C. Chase, and C. J. Chang-Hasnain, Opt. Express 18, 12606 (2010).

6. D. Fattal, J. Li, Z. Peng, M. Fiorentino, and R. G. Beausoleil, Nat. Photon. 4, 466 (2010).

7. D. Zhao, H. Yang, Z. Ma, and W. Zhou, Opt. Express 19, 9050 (2011).

8. H. Wu, W. Mo, J. Hou, D. Gao, R. Hao, R. Guo, W. Wu, and Z. Zhou, J. Opt. 12, 015703 (2010).

9. W.-M. Ye, X.-D. Yuan, C.-C. Guo, and C. Zen, Opt. Express 18, 7590 (2010).

10. L. Carletti, R. Malureanu, J. Mørk, and I.-S. Chung, Opt. Express 19, 23567 (2011).

11. V. Karagodsky, F. G. Sedgwick, and C. J. Chang-Hasnain, Opt. Express 18, 16973 (2010).

12. M. G. Moharam, E. B. Grann, D. A. Pommet, and T. K. Gaylord, J. Opt. Soc. Am. A 12, 1068 (1995).

13. M. Mutlu, A. E. Akosman, A. E. Serebryannikov, and E. Ozbay, Opt. Lett. 36, 1653 (2011).

14. E. D. Palik, Handbook of Optical Constants of Solids (Academic, 1998).

15. C. A. Balanis, Antenna Theory: Analysis and Design (Wiley, 2005). 